\title{
Comparative analysis of flax (Linum usitatissimum L.) genomes and transcriptomes
}

\author{
Elena Pushkova \\ Engelhardt Institute of Molecular \\ Biology, RAS, Moscow, Russia \\ pushkova18@gmail.com
}

Liubov Povkhova

Engelhardt Institute of Molecular Biology, RAS, Moscow, Russia;

Moscow Institute of Physics and

Technology, Dolgoprudny, Russia povhova.1v@phystech.edu

Tatiana Rozhmina

Engelhardt Institute of Molecular Biology, RAS, Moscow, Russia; Federal Research Center for Bast Fiber Crops, Torzhok, Russia

tatyana_rozhmina@mail.ru

\author{
George Krasnov \\ Engelhardt Institute of Molecular \\ Biology, RAS, Moscow, Russia \\ gskrasnov@mail.ru
}

Artemy Beniaminov

Engelhardt Institute of Molecular Biology, RAS, Moscow, Russia abeniaminov@mail.ru

Alexey Dmitriev

Engelhardt Institute of Molecular Biology, RAS, Moscow, Russia alex_245@mail.ru

\author{
Roman Novakovskiy \\ Engelhardt Institute of Molecular \\ Biology, RAS, Moscow, Russia \\ 0legovich46@mail.ru
}

Nadezhda Bolsheva

Engelhardt Institute of Molecular Biology, RAS, Moscow, Russia

nlbolsheva@mail.ru

Nataliya Melnikova

Engelhardt Institute of Molecular Biology, RAS, Moscow, Russia mnv-4529264@yandex.ru

\begin{abstract}
Flax (Linum usitatissimum L.) is an agriculturally important plant that has a wide range of applications in industry, and the direction of cultivar application is determined by its genetic characteristics. The present work aimed to obtain high-quality sequences of flax genomes and transcriptomes for genetically diverse cultivars and lines, which have breeding value and different direction of use. Six cultivars/lines (LM98, \#3896, Diplomat, Atlant, Universal, Alizee) were selected for the present work. We have developed an optimal method for the extraction of pure highmolecular-weight DNA from flax plants and performed genome and transcriptome sequencing. From 6 to $10 \mathrm{~Gb}$ was obtained for each of the studied flax cultivars/lines on the Oxford Nanopore platform and 20-25 million reads on the Illumina platform. Transcriptome sequencing of different flax tissues was also performed. Genome assembly using Flye resulted in the N50 value from $200 \mathrm{~kb}$ to $1 \mathrm{Mb}$ depending on the genotype. Genome annotation was then performed. The obtained genome assemblies are the basis for molecular genetic studies in flax and allow assessment of the differences in $L$. usitatissimum cultivars/lines at the genome-wide level.
\end{abstract}

Keywords - flax, genome assembly, transcriptomes, Nanopore sequencing

\section{Motivation and aim}

\section{Motivation}

The data on the nucleotide sequences of whole genomes and transcriptomes are the basis for molecular genetic studies in plants, including the analysis of gene expression, polymorphism assessment, development of molecular markers of economically valuable traits, progress in genome selection, and the use of CRISPR/Cas genome editing technologies. The revolution in DNA sequencing technologies that took place in recent decades allowed us to take a study of plant genomes and transcriptomes to a new level and to determine the genome sequences for thousands of genotypes of various species, as well as to resequence genomes for different genotypes of the same species [1]. However, as the number of sequenced plant genomes increases, it becomes clear that there are differences between genotypes of a single species in both single nucleotide polymorphisms and copy number of genes, the presence of extensive insertions/deletions and genome-specific loci, some of which are associated with economically valuable traits [2-5]. Therefore, representative sets of samples should be analyzed to obtain comprehensive data concerning genomes and transcriptomes within the studied species. For the present study, such agriculturally important plant as flax (Linum usitatissimum L.) was chosen. Flax seeds are the richest source of omega-3 fatty acids, which reduce the risk of cancer and cardiovascular diseases, and lignans, which have antibacterial, antifungicide, antioxidant, and anti-carcinogenic activity, and also contain easily digestible proteins, dietary fiber, vitamins, and minerals. Flax fiber is used in the production of highquality textiles, medical devices, and composite materials. Thus, flax has multiple using in different areas and the direction of cultivar application is determined by its genetic characteristics.

\section{Aim}

The aim of the present work was to obtain high-quality sequences of flax genomes and transcriptomes for genetically diverse cultivars and lines, which have breeding value and different direction of use.

\section{Methods}

When choosing flax genotypes for comparative analysis of genomes and transcriptomes, we took into account their genetic differences, resistance to the most harmful stresses, namely the Fusarium oxysporum fungus and adverse soil acidity, and other economically significant characteristics, such as yield, oil composition, fiber quality. As a result of joint research with the Institute for Flax (Torzhok), 6 cultivars/lines were selected (LM98, \#3896, Diplomat, Atlant, Universal, Alizee) that differ in valuable traits and are of interest for use in creating improved varieties. To obtain a high-quality assembly of flax genomes, an approach was chosen using a combination of two sequencing platforms, one of which (Oxford Nanopore) allows for reads of large length but insufficient accuracy, and the other (Illumina) - for high- 
precision but short reads. For sequencing on the Oxford Nanopore platform, an important task was the isolation of high-quality DNA. We tested numerous approaches, and as a result, developed an optimal method for the extraction of pure high-molecular-weight DNA from flax. The method was based on obtaining the maximum yield of high molecular weight but not very pure DNA with the DNA-EXTRAN-3 (Synthol) kit and the further purification, including precipitation with CTAB containing buffer and the using of ion-exchange columns from the Blood \& Cell Culture DNA Mini Kit (Qiagen). HiSeq 2500 (Illumina) and MinION (Oxford Nanopore) were used for sequencing genomes of six selected flax cultivars/lines. NextSeq (Illumina) was used for sequencing the transcriptomes of the roots and shoots of 7 day-old seedlings, leaves and stems of 6-week-old plants, as well as flowers of LM98, \#3896, Diplomat, Atlant, Universal, Alizee cultivars/lines (in total, 30 cDNA libraries). For genome assembly, Shasta, wtdbg2, and Flye were used. The polishing of assemblies was performed using Racon and Pilon, and funannotate was used for genome annotation.

\section{Results}

From 20 to 25 million reads with a length of $250+250 \mathrm{bp}$ was obtained on the Illumina platform for each of the studied flax cultivar/line (LM98, \#3896, Diplomat, Atlant, Universal, Alizee), which after trimming corresponded to 20-25-fold coverage of the flax genome, and from 6 to $10 \mathrm{~Gb}$ was obtained on the Oxford Nanopore platform, which corresponds to 15-25-fold genome coverage. From 6 to 16 million reads with a length of 86 bases were received as a result of transcriptome sequencing of the roots and shoots of 7-day-old seedlings, leaves, and stems of 6-week-old plants, as well as flowers of LM98, \#3896, Diplomat, Atlant, Universal, Alizee cultivars/lines. Three different assemblers (Shasta, wtdbg2, and Flye) were compared to select the optimal strategy for flax genome assembly. The best results were shown by Flye. Then the polishing of assemblies was performed in two stages - using Nanopore reads (Racon) and using Illumina reads (Pilon). The N50 value ranged from 200 $\mathrm{kb}$ to $1 \mathrm{Mb}$, depending on the genotype, which is a good result. The completeness of the assemblies was more than $90 \%$ according to BUSCO metrics. The key point for the assembly quality was the length of the Oxford Nanopore reads - a lower output of reads but of a larger length allowed achieving a better quality of genome assembly. Using funannotate, genome annotation was performed. The obtained genome assemblies are the basis for molecular genetic studies in flax, allows one assessment of the differences in L. usitatissimum cultivars/lines at the genome-wide level, and lay the foundation for the development and introduction of markerassisted and genomic selection of flax and its genome editing. Based on transcriptome sequencing data, gene expression was also evaluated in different tissues of six sequenced cultivars/lines, and genes with the most significant differences in expression between flax genotypes with different characteristics were revealed that is important for identifying associations between gene expression and phenotype.

\section{Acknowledgment}

This work was financially supported by the Russian Science Foundation, grant 16-16-00114.

\section{References}

[1] Torkamaneh, D., B. Boyle, and F. Belzile, Efficient genome-wide genotyping strategies and data integration in crop plants. Theor Appl Genet, 2018. 131(3): p. 499-511

[2] Thudi, M., et al., Whole genome re-sequencing reveals genome-wide variations among parental lines of 16 mapping populations in chickpea (Cicer arietinum L.). BMC Plant Biol, 2016. 16 Suppl 1: p. 10.

[3] Bayer, P.E., et al., Assembly and comparison of two closely related Brassica napus genomes. Plant Biotechnol J, 2017. 15(12): p. 16021610 .

[4] Jiao, Y., et al., Improved maize reference genome with single-molecule technologies. Nature, 2017. 546(7659): p. 524-527.

[5] Schatz, M.C., et al., Whole genome de novo assemblies of three divergent strains of rice, Oryza sativa, document novel gene space of aus and indica. Genome Biol, 2014. 15(11): p. 506. 